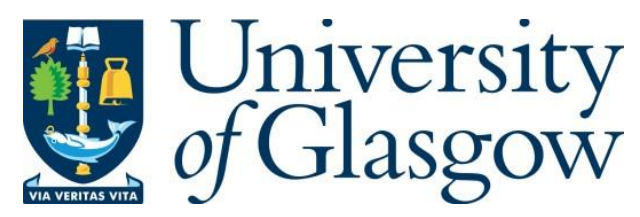

Ashleibta, A. M., Abbasi, Q. H., Shah, S. A., Khalid, A., AbuAli, N. A. and Imran, M.

A. (2020) Non-invasive RF sensing for detecting breathing abnormalities using software defined radios. IEEE Sensors Journal, (doi: 10.1109/JSEN.2020.3035960).

There may be differences between this version and the published version. You are advised to consult the publisher's version if you wish to cite from it.

http://eprints.gla.ac.uk/225797/

Deposited on: 6 November 2020

Enlighten - Research publications by members of the University of Glasgow http://eprints.gla.ac.uk 


\title{
Non-Invasive RF Sensing for Detecting Breathing Abnormalities using Software Defined Radios
}

\author{
Aboajeila Milad Ashleibta, Qammer H. Abbasi, Senior Member, IEEE, Syed Aziz Shah, Arslan Khalid, \\ Najah Abed AbuAli, Senior Member, IEEE and Muhammad Ali Imran, Senior Member, IEEE,
}

\begin{abstract}
The non-contact continuous monitoring of biomarkers comprising breathing detection and heart rate are essential vital signs to evaluate the general physical health of a patient. As compared to existing methods that need dedicated equipment (such as wearable sensors), the radio frequency (RF) signals can be synthesised to continuously monitor breathing rate in a contact-less setting. In this paper, we proposed the contact less breathing rate detection using universal software radio peripheral (USRP) platform without any wearable sensor. Our system leverage on the channel state information (CSI) to record the minute movement caused by breathing over orthogonal frequency division multiplexing (OFDM) in multiple sub-carriers. We presented a comparison of our breathing rate detection with wearable sensor (ground truth) results for single human subject. In this paper, we used wireless data to train, validate and test different machine learning $(\mathrm{ML})$ algorithms to classify USRP data into normal, shallow and elevated breathing depending on the breathing rate. Although different ML models were developed using the K-Nearest Neighbor (KNN), Discriminant Analysis (DA), Naive Bayes (NB) and Decision Tree (DT) algorithms, however results showed KNN based model provided the highest accuracy for our data (91\%) each time the trial was made. DT (17.131\%), DA (59.72\%) and NB (48.99\%). Results presented in this paper showed that USRP based breathing rate is comparable to the wearable sensor demonstrating the potential application of our method to accurately monitor breathing rate of patients in primary or acute setting.
\end{abstract}

Index Terms - Vital Signs, university software radio peripherals USRPs, channel state information, software defined radios, healthcare application.

\section{INTRODUCTION}

$\mathbf{T}$ HERE is a growing interest in the development of universal, contact-less and wireless sensing technologies to monitor daily activities. Several approaches for tracking human health and vital signs including breathing detection have been developed recently. Some of them include the use of smartphones and wearable sensor [1], [2], doppler radar [3], [4], Wi-Fi [5], ultra-wideband radar [6] and frequency modulated carriers [7]. Traditional techniques such as the received signal strength (RSS) provide a single frequency carrier power measurement with regards to full available

Ashleibta is with James Watt School of Engineering, University of Glasgow, Glasgow,G12 8QQ, U.K.(E-mail: 2449786a@ student.gla.ac.uk

Syed Aziz Shah, Associate Professor of Mobile Health. Centre for Intelligant Healthcare, Coventry University CV1 5FB U.K. (E-mail: ad5190@coventry.ac.uk).

Najah AbuAli, Professor at the Faculty of Information Technology in the United Arab Emirates University (UAEU), UAE (E-mail: najah@uaeu.ac.ae).

Arslan Khalid and Muhammad. A. Imran and Qammer. H. Abbasi are with James Watt School of Engineering, University of Glasgow, Glasgow, G12 8QQ, U.K. ( Muhammad.Khalid, Muhammad.Imran and Qammer.Abbasi@glasgow.ac.uk). frequency bandwidth. Amongst other traditional ways methods to monitor vital signs include wearable sensors, accelerometers (e.g. smartphones) or imaging sensors [8], [9]. Wearable sensor and accelerometers require physical contact with the body and have particularly slow response time whereas the imaging sensors are parasitical of particularity [10] and have issues to penetrate through walls, and darkness [11]. Radio frequency (RF) based systems offer non-invasive breathing rate monitoring through analysis of RF signal reflected off the human body. RF based monitoring systems which use frequency modulated continuous wave radar [12], [13] or doppler radar [4] are complex and expensive. Breath-tracking using RSS wireless devices are discussed in [15], have limited bandwidth around $7 \mathrm{Ghz}$ and $60 \mathrm{Ghz}$ millimetre wave signal which leads to false detection in long distance and require high gain directional antenna to covered. Mechanisms such as the USRP, frequency-modulated continuous-wave (FMCW), radar sensor and passive radar sensor [12], [13], [14] offer lowcost, long-term non-invasive breathing rate monitoring without requiring hospital visits or a physical contact. However, these systems need dedicated software and hardware setup operated at higher frequency range. 
Recent work by Patwari et al. [15] and Kaltiokallio et al. [16] used RSS and CSI data obtained using Wi-Fi signals to detect human breathing. This approach requires extra wireless sensor nodes to be deployed in indoor settings. Another study by Nasser, et al. [17] utilised WiFi RSS for breathing rate estimation and is capable of monitoring breathing for multiple people in parallel. Channel state information (CSI) is temporally more stable as compared to RSS as it presents both amplitude information and phase information. Recently, Wang, et al. [18] exploited CSI phase differences from the Wi-Fi router. The channel state information based wireless sensing systems have been also used for detecting respiration rate of people. For example, the author in [19] shown breathing detection based on CSI that extracted from WiFi. The achieved accuracy of this system reaches $94 \%$. Moreover, the work shown in [21] use the CSI amplitude information to measure respirator rate when the person is asleep, this system requires the person to remain asleep. CSI based detection systems have numerous advantages; they do not require any wearable devices and also preserve user privacy. Although, the CSIbased method is efficient for activity detection in indoor settings, the main limitation of using Wi-Fi routers is their scalability, flexibility, and under-reporting of subcarriers. Network interface card (NIC) used by CSI systems only exploit 30 subcarriers and not revealing all 56 frequency channels transmitted by Wi-Fi router which accounts for $42 \%$ of loss in frequency carriers. Also, NIC based systems do not have the flexibility to increase/decrease frequency subcarriers, power level of transmitter signal, remove noise, and change operating frequency [22], [20].

By extracting the CSI data using USRPs transceiver model, our system allows custom configuration transmitted and received power and the operating frequency swing. In addition, it offers easy implementation of signal processing algorithms and the ability to reuse hardware (e.g. self-designed antennas). In this paper, we propose a flexible and scalable wireless sensing driven by USRP in conjunction with machine learning algorithm to detect human breathing. Our system designed using a MATLAB Simulink model, exploits 64 OFDM subcarriers to receive all the transmitted sub-carriers and detect breathing rate of single person. Thy system we have proposed overcomes all these challenges as USRP operating frequency is flexible, number of subcarriers can be changed in real time with low-noise figure. We have used USRP by transmitting and receiving $\mathrm{N}$ number of multiple OFDM subcarriers as compared to the counterpart where only limited numbers are available. Our algorithms provide real-time classification on the collected data from human breathing activities and high-classification accuracy for empirical results. The results obtained using USRP based wireless sensing for activities of respiration are highly accurate as compared to off-the-shelf wireless devices each time when activities and experiments are performed. Our system can also be used to detect large scale body movements of a person.

In the rest of this paper, we explain the design of SDR based human activity detection platform and signal propagation. We describe the experimental setup and system parameter and evaluate the performance of the system followed by the conclusion.

\section{A. Contribution and Novelty}

Numerous studies have demonstrated CSI based on Wi-Fi signal to recognise human movement exploiting lowcost small wireless devices such as Wi-Fi router, network interface card [20][22]. The main limitation of using these devices is the scalability, flexibility, and under-reporting all group of subcarriers. The Wi-Fi sensing for human activity recognition only report limited number of sbucarriers. The software defined radio allows us to change various parameters such as number of frequency carriers, power level and radiation pattern in realtime. In addition, systems based on SDR are also flexible, scalable and delivers desired result reliably and accurately.

\section{METHOD}

In this part, we discussed the design of simulink project and generating RF signal through the USRP.

\section{A. System Overview}

In the transmitter operation, we designed a MATLAB Simulink program to generate the OFDM signal for transmitter and receiver process over multiple subcarriers based on universal software radio peripheral USRPX300. Random data bits were generated from work space with probability of 0.75 . then QPSK modulation scheme were carried out to convert these bits to symbols, each two bits represent one symbol. Then the symbols were connected to single subcarrier are plugged into the converter to transform it serial to parallel to by applying Inverse Fast Fourier Transform (IFFT) operation and transfer the symbols from frequency domain to time domain. After that a cyclic prefix are then given into the system between each symbol to mitigate the effect of co-channel interference of the collected signal at the receiver side. Then the received signal is up-converted through Digital-to-Analog converter (DAC) using USRP platform.

At the receiver end, the OFDM signal was collected by the received antenna that equipped on the second USRPX310, then the USRP convert the OFDM signal from analogue to digital and next down converting it to the original base band signal, Afterwards a low-pass filter was used in the USRP to generate the I/Q base-band wave form and remove the effect of high frequencies. Besides, OFDM bits are normally sorted into frames so that the received signal needs to be synchronised in time and frequency to obtain the start of the OFDM symbol and then Fast Fourier Transform (FFT) algorithm used to convert the based-band signal from time to frequency domain. Also, the guard interval removed through retrieving the original signal. The block diagram of the transceiver operation can be shown in figures 1 .

\section{B. Signal Model}

The proposed system used CSI signal for the transmitter and receiver operation. The CSI represent the channel frequency response (CFR) for each OFDM subcarrier includes amplitude 


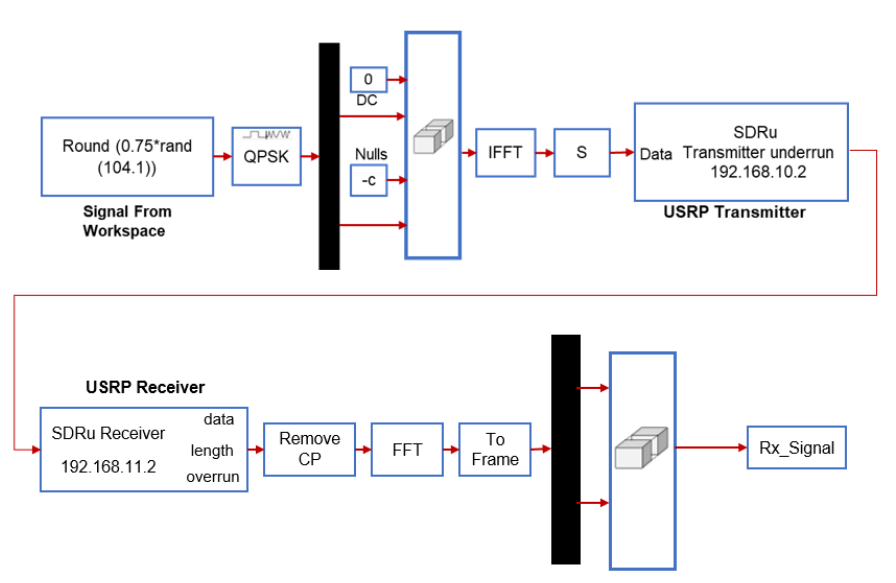

Fig. 1. Simplified OFDM Simulink Model for Transceiver Operation.

information and phase information. This system has been developed and implemented only to extract the wireless channel state information amplitude response for human activities. The CSI phase information is inapplicable due to presence of random noise, that is why it was not considered. Equation (1) presented the received CSI signal.

$$
H\left(f_{i}\right)=H\left(f_{i}\right) e^{j \angle H\left(f_{i}\right)}
$$

In this expression, $\mathrm{H}\left(f_{i}\right)$ describes the information of amplitude and $\angle \mathrm{H}\left(f_{i}\right)$ explains the phase information for CSI signal. The measured OFDM subcarriers contain the values of CSI packets and it can be shown in equation (2). [23].

$$
\mathrm{H}=\left(h_{1}, h_{2}, h_{3}, \ldots \ldots, h_{N}\right)
$$

In this system, IFFT and FFT blocks measure the response of amplitude and phase. By performing IFFT at transmitter side and FFT at receiver side. The channel response can be expressed as following. [24].

$$
H(f)=\frac{x(f)}{Y(f)}
$$

Where $\mathrm{X}(f)$ refers to the response of the OFDM transmitted signal, $\mathrm{H}(f)$ describe the system channel response and $\mathrm{Y}(f)$ represent the response of the OFDM received signal.

\section{Collection of data}

The collection of three breathing events carried out in lab environment using single subject. The experiment consists of two USRPs X300/310 and the distance between the subject and USRPs antenna keep it as 0.4 meters. The volunteer started breathing normal, Shallow, and heavy and the reflected signal from human body once performing breathing activities is stored as wireless channel state information WCSI data.

\section{Data extraction}

At the receiver side. The OFDM signal utilized for fine grained wireless channel state information extraction then the amplitude frequency response for each breathing activity will

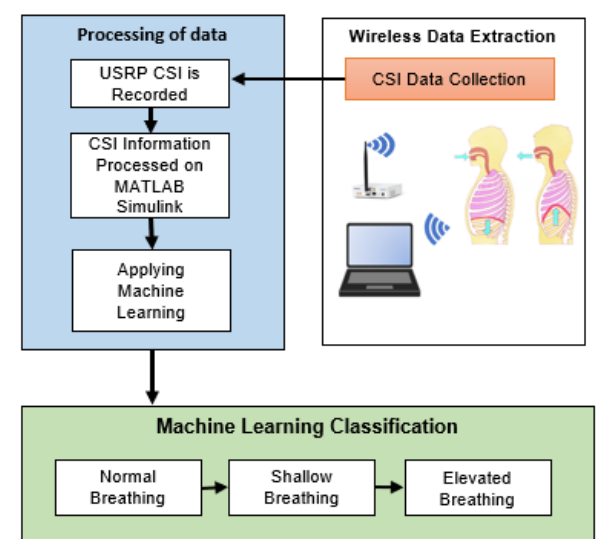

Fig. 2. Flowchart of the proposed System for collecting channel state information using USRPs.

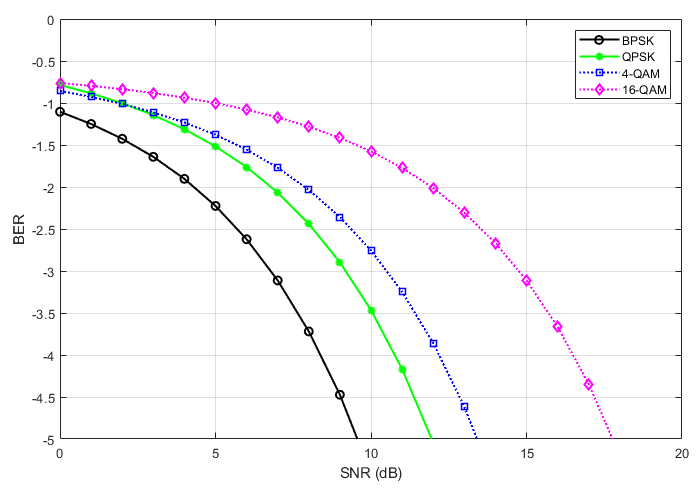

Fig. 3. Simulated Bit error rate analysis of OFDM system.

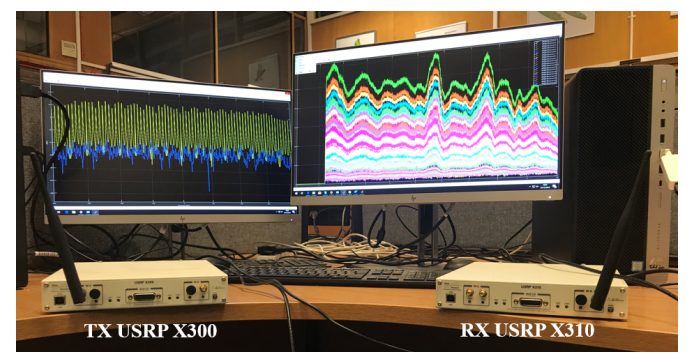

Fig. 4. Hardware design of System Setup .

be observed constantly for 10 seconds. And each collected signal consists several OFDM samples and subcarriers. Time and samples can be represented as the received number of samples in a unit time.

The obtained data from CSI is in the row form and needs processing to provide meaningful information using three following steps.

I- Cleaning the data by eliminating the terrible CSI data.

II- Applying low pass filter for removing the noise.

III- The grouping technique is performed to detect the correlation between the CSI values. 


\section{E. Additive White Gaussian Noise}

The Additive white Gaussian noise channel (AWGN) was considered for simulated result. This wireless medium model has been widely exploited in identifying the most feasible modulation scheme. The main advantages of this wireless channel model is it's least complexity in terms of test and deployment and represents the real man-made noise with regards to other multi-user interference [25].

\section{F. Bit Error Rate of the USRP Model}

Bit error rate (BER) gives the number of bits in error per unit time and the most important parameters to analyse the performance of any robust, efficient and accurate wireless medium system. Figure 3 shows BER versus SNR (Eb/No in $\mathrm{dB})$ performance analysis of various modulation techniques. The additive white gaussian noise wireless channel was used to obtain simulated results. It is clear from the figure that BPSK has lower BER than QPSK and QAM. For instance, at SNR 10 BER for BPSK is 0 but for QPSK and 16 QAM is greater than $10^{-4}$. Also, at SNR 14 BER for QPSK is 0 whereas for 16 QAM is approximate $10^{-3}$. Therefore, Quadrature amplitude modulation (16-QAM) has greater Bit Error Rate (BER) as compared with lower order Binary Phase Shfit Keying (BPSK). This is also proven for our proposed model as well.

\section{G. Feature extraction}

CSI information represent fine-grained data and feature extraction is used for transferring the CSI data into meaningful information. Several feature in the time domain are implemented. (1) the normalized standard division indicate the dispersion degree among sampling points of the CSI signal. (2) the root mean square (RMS) is used to calculate the magnitude of CSI information. (3) peak to peak value is used to calculate the differences between the maximum and minimum amplitudes values of the collected CSI signal. (4) the peak factor indicates if there is influence on the CSI information. (5) waveform factor is used to represent the ratio of the root mean squire value to the average value. (6) FFT is used to excerpt the frequency component with peak to peak values of the signal. (7) spectrum probability and signal energy are unique and essential for extraction of frequency domain analysis.

\section{EVALUATION}

Our experiments were carried out to test human vital signs monitor the respiratory rates and identify anomalies. This section describes the hardware and software setup involves the parameters used in the designed system.

\section{A. Experimental method}

The experiment is performed in lab environment using two NI USRPX300/310 platforms that have been built for real time data acquisition. The USRPs is fitted with two omni directional antennas for the transmit and receive operation. The antenna has frequency range from $2.4 \mathrm{GHz}$ to $5.9 \mathrm{GHz}$. The key advantage of using omni-directional antenna is that it can detect human activities in LOS and NLOS. Besides, directional antennas, yagi antennas have also been tested on our system and provided similar results.the experiment was conducted at $5.32 \mathrm{GHz}$, With the increase in frequency, the range resolution increases and vice versa. The respiratory rate will be best detected when the USRP transceiver model is tuned at higher frequencies. However, the distance in terms of monitoring person will be decreases and signal will be highly susceptible to external noise. Furthermore, two PCs was used to implement the trial and a 1 GB Ethernet cable was used to transmit the data to the centralize personal computer to process the acquired USRP data and classify normal respiratory rate against abnormal breathing rate. The trial were processed using MATLAB SIMULINK software. For the demonstration, the experiment carried out in lab environment, which was a large room with office environment. Also, the volunteer was positioned $0.4 \mathrm{~m}$ away from the antenna, in order to achieve optimum performance. The experiment was undertaken to capture the changes occurred in CSI data due to chest movement cause by respiratory patterns. Also, we used wearable sensor (Ground truth) to ensure that the collected data from USRP is working properly.The breathing sensor used as a reference was SA9311M - manufactured by Though Tech. The reference wearable sensor is a highly sensitive to chest movements and abdominal expansion/contraction and outputs the respiration waveform. Finally, four algorithms such as KNN, DA, NB and DT were applied to process and classify the collected data. In the same scenario, we have performed experimental campaign ask the volunteers to perform normal breathing, shallow breathing, and elevated breathing while they were under test. the experimental setup is shown in figure 4.

TABLE I

Software Configuration and Parameters Selection

\begin{tabular}{||ll||}
\hline Parameters & Values \\
\hline Input random bits & round $\left(0.75^{*}\right.$ rand $\left.(104,1)\right)$ \\
\hline Sample time rate & $132 / 104 *(1 / 132 \mathrm{e} 4)$ \\
\hline Modulation type & QPSK \\
\hline Bit per symbol M & 2 bits \\
\hline Used Subcarrier & 64 subcarriers \\
\hline Used Null subcarrier & 12 \\
\hline Used Pilot subcarrier & 4 \\
\hline Samples per frame & Used subcarrier $\log 2(\mathrm{M})$ \\
\hline NFFTpoints & $64,128,256,512,1024$ and 2048 \\
\hline Cycle prefix & NFFT-data subcarrier \\
\hline
\end{tabular}

\section{B. System Parameters Selection}

In this part, we introduce the system parameters selected for software and hardware system setup. Firstly, Simulink model was implemented in MATLAB based on QPSK modulation scheme and OFDM signal. The parameters values used in our software as shown in Table I. We tested the hardware parameters by running QPSK transmitter and receiver examples on MATLAB, then we applied our own hardware parameters of the USRPs X300/310 to capture the wireless CSI of small body movement. The trial was conducted at $5.32 \mathrm{GHz}$ for USRP platform and the sample rate chosen in this experiment was 80 . Configuration for the hardware parameters is shown in Table II. 
TABLE II

HaRdware Configuration and Parameters Selection

\begin{tabular}{||ll||}
\hline Platform & USRP X300/310 \\
\hline \hline TX serial number & 192.168 .10 .1 \\
\hline RX serial number & 192.168 .11 .1 \\
\hline Channel mapping & 1,2 \\
\hline Gain (dB) & 70 \\
\hline Master clock rate & $120 \mathrm{Mhz}$ \\
\hline Centre frequency of USRP & $5.32 \mathrm{Ghz}$ \\
\hline Sample time rate & $1 / 80 \mathrm{e} 4$ \\
\hline Local oscillator offset & Dialog \\
\hline Interpolation factor & 500 \\
\hline PPS source & Internal \\
\hline Decimation factor & 500 \\
\hline Clock source & Internal \\
\hline Transport data type & Int16 \\
\hline
\end{tabular}

\section{Results And Discussions}

In this section, we evaluated the overall performance of the breathing rate under three different regimes; normal breathing, shallow breathing, and elevated breathing. The three breathing regimes were measured by two devices USRPs and wearable sensor (ground-truth) after acquiring ethical approvals from University of Glasgow. Firstly, the volunteer was asked to breath normally followed by shallow breathing and the elevated breathing for 10 seconds each. We collected the data from sensor and the USRP simultaneously. The wearable sensor was attached to the participant body's chest. While testing our experiment and recording of the data, multiple factors were considered in real-time environment such as the physical objects that could affect the wireless received signal. First, we tested our system with QPSK transmitter and receiver examples with USRP to ensuring that there is no error acquired for the device configuration and whether working properly. Then we used our own Simulink model to captivate the wireless signal for small scale body movement of the human. The number of packets characterise the number of subcarriers of the OFDM signal. Figure 5 shows Wireless channel state information (WCSI) waveform of normal breathing for both USRP and wearable sensor.We have used The Breathing Rate Belt and Pressure Sensor 'product code 3190 as reference sensor. The human started sitting in front of the USRP and kept the distance as 0.4 meters. Also covered the sensor around his body and started breathing normally and recorded the data of both the USRP and sensor. We noticed that the amplitude changes were normally based on the habitual breathing of the human. In this case, the practice was repeated several times with same amplitude differences detected.

During recording of the wireless data from human respiration, we set the time to 10 seconds. Transmitted packets were 10000 and received 8642 out of 10000 packets for 10 second time duration. We repeated the normal breathing activity 10 times and received same number of packets of each activity performed. We used the wearable sensor as reference. As the measured data of wearable sensor are reliable and presents the ground truth of the breathing detection. The wireless value is close to the wearable value. This ensures that the system can measure the human breathing without wearing any sensor and it can be alternative of wearable devices. Figure 6. shows the results when of shallow breathing. The figure illustrates the prominent changes in the amplitude. The time duration in this practice was also set for 10 seconds including the same number of send and received packets of the previous activity of normal breath. In this work, we have used software-defined-radio model University Software Radio Peripherals (USRP) by transmitting and receiving $\mathrm{N}$ number of multiple OFDM subcarriers as compared to its counterpart where limited numbers are available. In our experiment, The Orthogonal Frequency Division Multiplexing (OFDM) with 64-subcarriers is used to extract the Wireless Channel State Information of breathing activities.

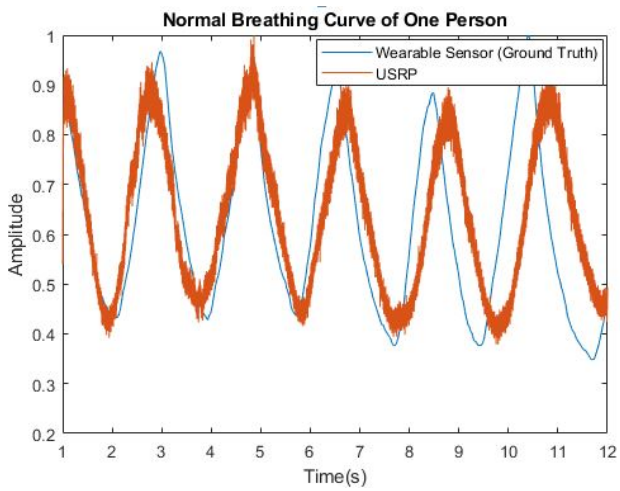

Fig. 5. WCSI during Normal Breathing for USRP and Sensor.

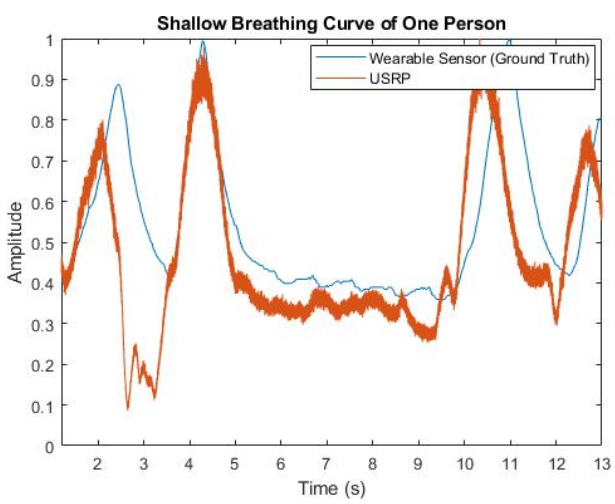

Fig. 6. WCSI during shallow Breathing for USRP and Sensor.

Figure 7 represents the results of the elevated breathing. It can be seen that the signal increased in the cycle of the amplitude compared to the waveform of normal breathing. Also, comprised the similar number of the transmitted and received packets for 10 seconds time duration. Besides, the referenced signal is reliable and has less noise compared with the signal obtained from the SDR or is effected by any physical factors as a result of performing the breathing activities.

\section{A. Machine Learning Classification}

This section provided details on the discussion of four different machine learning algorithms used to classify three breathing events and evaluate the suggested system based on percentage accuracy. The dataset performance was obtained 
TABLE III

FEATURE EXTRACTION EQUATIONS FOR DATA CLASSIFICATION

\begin{tabular}{|c||c||c|}
\hline NO & Feature & Expression \\
\hline 1 & normalized standard division & $Y_{s d}=\sqrt{\frac{1}{N-1} \sum_{i=1}^{N}\left(\mathrm{x}_{i}-y_{m}\right)^{2}}$ \\
\hline 2 & the root mean square (RMS) & $Y_{R M S}=\sqrt{\frac{1}{N} \sum_{i=1}^{N} \mathrm{x}_{i}{ }^{2}}$ \\
\hline 3 & Peak to peak value & $Y_{p p v}=Y_{\max }-Y_{\min }(i=1,2 \ldots, N)$ \\
\hline 4 & Peak factor & $Y_{P}=\frac{\max \left(x_{i}\right)}{Y_{R M S}}(i=1,2 \ldots, N)$ \\
\hline 5 & Waveform factor & $Y_{w}=\frac{N_{*} Y_{R M S}}{\sum_{i=1}^{N} X_{i}}(i=1,2 \ldots, N)$ \\
\hline 6 & FFT & $Y_{F F T}=\sum_{n=-N}^{N} \mathrm{x}(\mathrm{n}) e^{-j \frac{2 \pi}{N} n d}$ \\
\hline 7 & Spectrum probability & $Y_{S E}=\sum_{n=-N}^{N} \mathrm{p}(\mathrm{d})^{2}$ \\
\hline 8 & Signal energy & $Y_{M}=\sum_{n=-N}^{N} \mathrm{p}(\mathrm{d}) \ln \mathrm{p}(\mathrm{d})$ \\
\hline
\end{tabular}

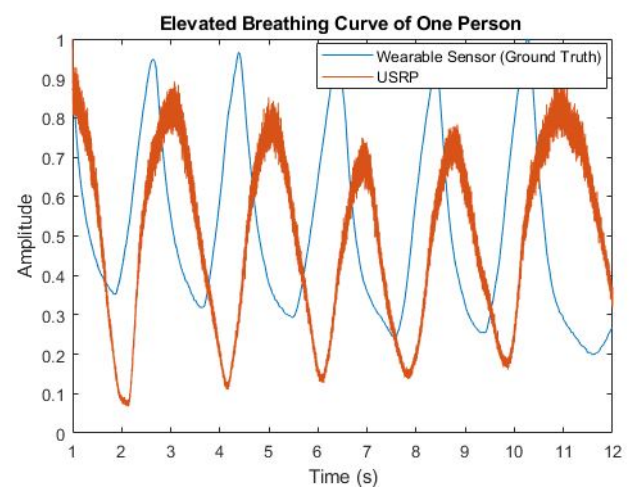

Fig. 7. WCSI during elevated breathing for USRP and Sensor.

using different ML techniques as listed above. A 10-fold cross validation technique was used on the USRP data containing different respiratory patterns.

The accuracy is calculated as an average of the 10 sets of testing data used in each of the 10 cross fold validation process. The below Figures 8-9 shows the confusion matrix of KNN and DT algorithms. It can be seen from Confusion matrix in figure 8-9 that the $y$ axis represents the predicted classes and $\mathrm{x}$ axis symbolizes the true class of the algorithm.

The machine learning algorithms were run using the following parameters. KNN is configured using $3 \mathrm{~K}$-samples using the Euclidean distance. Discriminant Analysis DA was configured as linear. Naive Bayes NB used the normal distribution method. DT algorithm is set up to use 50 splits in the decision tree.

The KNN classifier provide the best classification accuracy among other algorithms. The value for $\mathrm{KNN}$ algorithms was selected as 3 . The confusion matrix in figure 8 represent a total of 1075851 samples were received over a period for all breathing events. For a combined activity of elevated breath, $15.4598 \%$ were correctly classified as elevated breath. While $1.3388 \%$ samples were incorrectly classified as breath normal and $0.3538 \%$ samples as shallow breath activity.

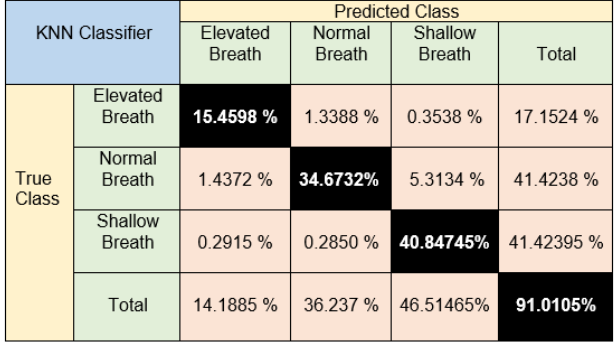

Fig. 8. Confusion matrix for KNN classifier.

TABLE IV

FOLLOWING PARAMETERS WERE USED FOR KNN AND DT CLASSIFIER

\begin{tabular}{||lll||}
\hline $\begin{array}{l}\text { Classification Al- } \\
\text { gorithm }\end{array}$ & Parameters & Setup \\
\hline \hline Decision Tree & $\begin{array}{l}\text { Maximum number of } \\
\text { dataset splits. Split }\end{array}$ & $\begin{array}{l}\text { 4 Gini's diversity in- } \\
\text { Criterion }\end{array}$ \\
& $\begin{array}{l}\text { Number r of } \\
\text { Neighbours. Distance }\end{array}$ & \\
\hline K-Nearest & 2 Euclidean \\
Neighbour & Metric & \\
\hline
\end{tabular}

Almost similar number of samples were correctly identified as breath normal and $1.4372 \%$ samples were determined as elevated breath besides $5.3134 \%$ CSI samples as shallow breath. $40.84745 \%$ samples were predicted as shallow breath while a combine of nearly $0.5765 \%$ were unclassified as other remaining activities. The overall percentage accuracy using KNN classifier was obtained as $91.0105 \%$

DT algorithm performed worse, providing overall accuracy of only $71.131 \%$. It can be seen in Figure 9 that for first activity, there were $5.7335 \%$ classified correctly. $4.5800 \%$ CSI samples were identified as breath normal activity (false negative) and $6.8390 \%$ samples as shallow breathing. The classification of normal breathing samples slightly more accurate. $1.4247 \%$ and $14.7483 \%$ samples were classified incorrectly as elevated breath and shallow breath, this leaves the remaining $25.2507 \%$ breath normal samples as being correctly classified. 


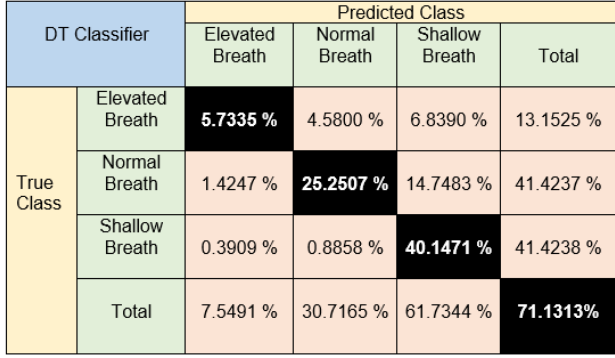

Fig. 9. Confusion matrix for DT classifier.

For the shallow breath activities, $40.1471 \%$ samples were classified correctly, then $0.3909 \%$ and $0.8858 \%$ samples were misclassified. The other algorithms tested were discriminant analysis (DA) and native bayes (NB) which produced poor results compared to KNN and DT algorithms with accuracy of only $59.72 \%$ for DA and $48.99 \%$ for NB. Table V shows the accuracy comparison for all used classifiers.

TABLE V

PERCENTAGE ACCURACIES OF EACH CLASSIFIER

\begin{tabular}{||ll||}
\hline Classifier models & Classification Accuracy \% \\
\hline \hline Nearest Neighbor (KNN) & 91.0105 \\
\hline Decision Tree (DT) & 71.131 \\
\hline Discriminant Analysis (DA) & 59.72 \\
\hline Naive Bayes (NB) & 48.99 \\
\hline
\end{tabular}

\section{CONCLUSION}

In this paper, we presented tracking breathing rate detection based on wireless signals. In particular, we designed a MATLAB Simulink model based on universal software radio peripheral (USRP) to capture small scale body movement. Our algorithms grounded on channel state information (CSI) information in time domain can detect the breathing rate for individual non invasively. In this work, we studied three breathing scenarios; normal, shallow and elevated breathing. We compared our results with a data harvesting sensor as the ground truth. Our results show high correlation between the two methods. Machine learning algorithm were applied for processing and classifying the data to provide excellent performance and robustness on breathing rate. For future work, we will increase complexity of the data collection by monitoring multiple people simultaneously, increase other movements in the surrounding and so on. our aim is to make this system more generalize, acquiring data in elderly care centre or hospitals in different geometrical settings. The CSI at heterogenous environment varies also, we will develop an algorithm for calibration in future work that in independent of geometrical structure.

\section{ACKNOWLEDGMENT}

Aboajeila Milad studentship is funded by Libyan Government. This work is supported in parts by Zayed Health Center at UAE University under Fund code G00003476, EPSRC EP/T021020/1 and EP/T021063/1.

\section{REFERENCES}

[1] D. Aranki, G. Kurillo, P. Yan, D. M. Liebovitz, R. Bajcsy, "RealTime Tele-Monitoring of Patients with Chronic Heart-Failure Using a Smartphone": Lessons Learned," IEEE Transactions on Affective Computing, vol. 7, pp. 206-219, 2016.

[2] T. Hilbel, S. Feilner, M. Struck, C. Hofmann, A. Heinig, H. A. Katus, "Cor/log BAN BT a wearable battery powered mHealth data logger and telemetry unit for multiple vital sign monitoring," 2016 Computing in Cardiology Conference (CinC), pp. 273-276, 2016.

[3] Droitcour, A. D., Boric-Lubecke, O., and Kovacs, G. T. Signal-tonoise ratio in doppler radar system for heart and respiratory rate measurements.MicrowaveTheoryandTechniques,IEEETrans.(2009).

[4] Fletcher, R., and Han, J. Low-cost differential front-end for doppler radar vital sign monitoring. In IEEE MTT (2009).

[5] Kaltiokallio, O. et. Al. Non-invasive respiration rate monitoring using a single cots tx-rx pair. In ACM/IEEE IPSN (2014).

[6] de Chazal, P., O'Hare, E., Fox, N., and Heneghan, C. Assessment of sleep/wake patterns using a non-contact biomotion sensor. In IEEE EMBS (2008).

[7] Balakrishnan, G., Durand, F., and Guttag, J. Detecting pulse from head motions in video. In IEEE CVPR (2013).

[8] C. A. Kushida, M. R. Littner, T. Morgenthaler, C. A. Alessi, D. Bailey, J. Coleman Jr, L. Friedman, M. Hirshkowitz, S. Kapen, M. Kramer et al., "Practice parameters for the indications for polysomnography and related procedures: an update for 2005," Sleep, vol. 28, no. 4, pp. 499-521, 2005.

[9] F. Adib, H. Mao, Z. Kabelac, D. Katabi, and R. C. Miller, "Smart Homes that Monitor Breathing and Heart Rate," CHI. ACM, pp 837-846, Seoul, Republic of Korea, April 18-23, 2015.

[10] Kelly caine et al. The effect of monitoring by camera and robots on the privacy enhancing behaviours of older adults. In Proc. of HRI, 2012.

[11] O. Kaltiokallio et al. Follow @grandma: Long-term device-free localization for residential monitoring. In Proc. of LCN Workshops, 2012.

[12] F. Adib, Z. Kabelac, and D. Katabi, "Multi-person motion tracking via RF body reflection ,MIT technical report,

[13] F. Adib, Z. Kabelac, H. Mao, D. Katabi, and R. C. Miller, "Demo: Realtime breath monitoring using wireless signals," in MobiCom, 2014.

[14] Wenda Li, Bo Tan and Robert J. Piechocki. Non-Contact Breathing Detection Using Passive Radar

[15] N.Patwari,, Wilson, J., Ananthanarayanan, S., Kasera, S., Westenskow, D. Monitoring Breathing via Signal Strength in Wireless Networks. IEEE Transactions On Mobile Computing, 1774-1786. doi: 10.1109/tmc.2013.117.2014.

[16] O. J. Kaltiokallio, H. Yigitler, R. J"antti, and N. Patwari, "Non-invasive respiration rate monitoring using a single cots tx-rx pair," in IPSN, 2014, pp. $59-70$.

[17] H. Abdelnasser, K. A. Harras, and M. Youssef. 2015. Ubibreathe: A Ubiquitous non-invasive WiFi-based Breathing Estimator. In Proc. IEEE MobiHoc'15. ACM, Hangzhou, China, 277-286.

[18] X. Wang, Yang, C., Mao, S. TensorBeat. ACM Transactions On Intelligent Systems And Technology, 9(1), 1-27. doi: 10.1145/3078855. 2017.

[19] Sangyoun Lee, Young-Deok Park, Young-Joo Suh. Design and Implementation of Monitoring System for Breathing and Heart Rate Pattern using WiFi Signals. e36net, ydpark, yjsuh@postech.ac.kr 978-1-53864790-5/18/2018

[20] S.A.Shah, S.A.; Ren, A.; Fan, D.; Zhang, Z.; Zhao, N.; Yang, X.; Luo, M.; Wang, W.; Hu, F.; Rehman, M.U.; Badarneh, O.S.; Abbasi, Q.H. Internet of Things for Sensing: A Case Study in the Healthcare System. Appl. Sci. 2018, 8, 508.2018.

C. A. Kushida, M. R. Littner, T. Morgenthaler, C. A. Alessi, D. Bailey, J. Coleman Jr, L. Friedman, M. Hirshkowitz, S. Kapen, M. Kramer et al., "Practice parameters for the indications for polysomnography and related procedures: an update for 2005," Sleep, vol. 28 , no. 4, pp. 499-521, 2005.

[21] J. Liu, Y. Wang, Y. Chen, J. Yang, X. Chen, J. Cheng, "Tracking Vital Signs During Sleep Leveraging Off-the-shelf WiFi," MobiHoc. ACM, pp 267-276, Hangzhou, China, June 22-25, 2015.

[22] S. A. Shah and F. Fioranelli, "RF Sensing Technologies for Assisted Daily Living in Healthcare: A Comprehensive Review," in IEEE Aerospace and Electronic Systems Magazine, vol. 34, no. 11, pp. 26-44, 1 Nov. 2019, doi: 10.1109/MAES.2019.2933971.2019.

[23] X. Yang, Shah SA, Ren A, et al. Freezing of gait detection considering leaky wave cable. IEEE Transactions on Antennas and Propagation. 2019,67(1):554-561. https://doi.org/10.1109/TAP. 2878081. 2018. 
[24] M. B.Khan, , Yang, X., Ren, A. , Al-Hababi, M. A. M., Zhao, N., Guan, L., Fan, D. and Shah, S. A. Design of software defined radios based platform for activity recognition. IEEE Access, 7, pp. 31083-31088.2019.

[25] N. Karimian. Design and Analysis of OFDM System for Powerline Based Communication. University of Central Lancashire. 2011.

[26] D. Haider et al., "An efficient monitoring of eclamptic seizures in wireless sensors networks," Comput. Electr. Eng., vol. 75, pp. 16-30, 2019.

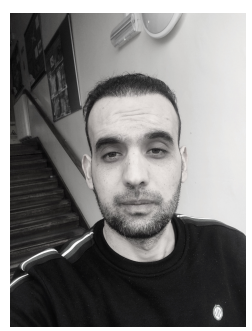

Aboajeila Milad Ashleibta was born in 1987 Banwalid, Libya. He has received his M.S. degree in electronic and electrical engineering from university of huddersfield. Untited kingdom in 2016. He is currently pursuing the $\mathrm{PhD}$ degree with university of Glasgow UK. His research interests involve wireless communication networks and electronics circuits.

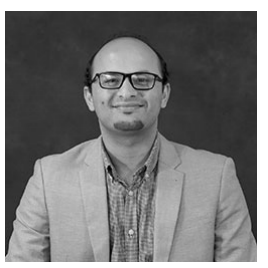

Qammer H. Abbasi (SM 16) received the B.Sc. and M.Sc. degrees in electronics and telecommunication engineering from the University of Engineering and Technology (UET), Lahore, Pakistan, and the Ph.D. degree in electronic and electrical engineering from the Queen Mary University of London (QMUL), U.K., in 2012. In 2012, he was a Postdoctoral Research Assistant with the Antenna and Electromagnetics Group, QMUL. He is currently a Senior Lecturer (Associate Professor) with the James Watt School of Engineering, University of Glasgow, U.K and researcher investigator with Scotland 5G Center. He has contributed to over 250 leading international technical journal and peer reviewed conference papers, and eight books. He received several recognitions for his research, which include appearance on BBC, STV, dawnnews, local and international newspapers, cover of MDPI journal, most downloaded articles, U.K. exceptional talent endorsement by Royal Academy of Engineering, National Talent Pool Award by Pakistan, International Young Scientist Award by NSFC China, URSI Young Scientist Award, National Interest Waiver by USA, four best paper awards, and best representative image of an outcome by QNRF. He is an Associate Editor for the IEEE JOURNAL OF ELECTROMAGNETICS, RF AND MICROWAVES IN MEDICINE AND BIOLOGY, the IEEE SENSORS JOURNAL, IEEE OPEN ACCESS ANTENNA AND PROPAGATION, IEEE ACCESS and acted as a guest editor for numerous special issues in top notch journals.

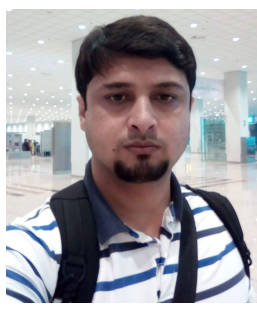

Syed Aziz Shah received the Ph.D. degree from Xidian University, China, in June 2018. He was a Postdoctorate Research Associate with the University of Glasgow and an Assistant Professor (lecturer) with Manchester Metropolitan University, U.K. He is currently Associate Professor of mobile health, centre for Intelligent Healthcare, Coventry University. He has (co)authored over 45 technical articles in top-rank crossdisciplinary journals, including IEEE and IET.His research interests include machine learning in wireless sensing, radar technology, software defined radios, antennas and propagation, and healthcare and agriculture technologies.

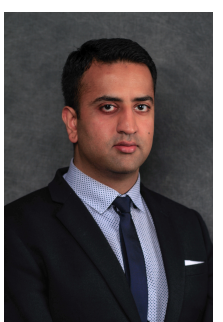

Muhammad Arslan Khalid holds a Ph.D. in Biomedical Engineering and a BEng. (Hons.) Electronics and Electrical Engineering from the University of Glasgow. Dr. Khalid specialised in the development of low-cost digital health and mobile health platforms working in close collaboration with University of California, Los Angeles. Dr. Khalid won numerous national entrepreneurship awards to commercialise a smartphonebased stroke diagnostic platform through a spinout company (iVisco), for which he also has three pending patents. He is currently a Healthcare Management Consultant at Deloitte and an affiliate at the James Watt School of Engineering, University of Glasgow.

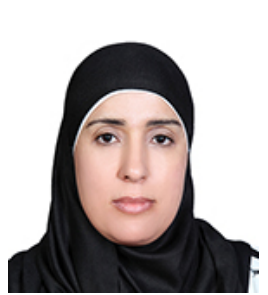

Najah Abu Ali is currently a Professor at the Faculty of Information Technology in the United Arab Emirates University (UAEU). She received her Ph.D. from the Department of Electrical and Computer Engineering at Queen's University in Kingston, Canada; specializing in resource management in computer networks. Her MSc and BSc were both attained in Electrical Engineering at the University of Jordan. Her general research interest includes modeling wireless communications, resource management in wired and wireless networks, and reducing the energy requirements in wireless sensor networks. More recently, she has strengthened her focus on the Internet of Things, particularly at the nano-scale communications, in addition, vehicle-to-vehicle networking. Her work has been consistently published in key publications venues for journals and conference. She's further co-authored a Wiley book on 4G and beyond cellular communication networks. She has also delivered various seminar and tutorials at flagship IEEE Communication Societies. She is an associate editor of the IEEE access Journal, She has also served as a reviewer for many noted journals, a TPC member and reviewer in several conferences (including ICC and Globecom), and contributed to the organization of several Sls and workshops. She also served as the Publication co-chair of IEEE GC'18.

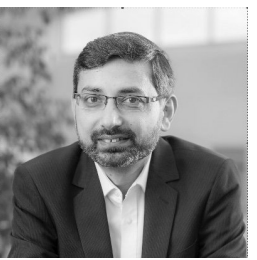

Muhammad Ali Imran (SM 12) received the M.Sc. (Hons.) and Ph.D. degrees from Imperial College London, U.K., in 2002 and 2007, respectively. He is Dean Glasgow College UESTC and a Professor of communication systems with the James WatSchool of Engineering, University of Glasgow. He is an Affiliate Professor at the University of Oklahoma, USA, and a Visiting Professor at the $5 \mathrm{G}$ Innovation Centre, University of Surrey, U.K. He is leading research in University of Glasgow for Scotland 5G Center. He has over 18 years of combined academic and industry experience, working primarily in the research areas of cellular communication systems. He has been awarded 15 patents, has authored/co-authored over 400 journal and conference publications, and has been principal/coprincipal investigator on over $£ 6$ million in sponsored research grants and contracts. He has supervised $40+$ successful Ph.D. graduates. $\mathrm{He}$ has an award of excellence in recognition of his academic achievements, conferred by the President of Pakistan. He was also awarded the IEEE Comsoc's Fred Ellersick Award 2014, the FEPS Learning and Teaching Award 2014, and the Sentinel of Science Award 2016. He was twice nominated for the Tony Jean's Inspirational Teaching Award. He is a shortlisted finalist for The Wharton-QS Stars Awards 2014, the QS Stars Reimagine Education Award 2016 for innovative teaching, and VC's Learning and Teaching Award from the University of Surrey. $\mathrm{He}$ is a Senior Fellow of the Higher Education Academy, U.K. He is the editor/coeditor of 8 books. 